ECCOMAS

Proceedia
EUROGEN 2021

$14^{\text {th }}$ ECCOMAS Thematic Conference on Evolutionary and Deterministic Methods for Design, Optimization and Control N. Gauger, K. Giannakoglou, M. Papadrakakis, J. Periaux (eds.) Streamed from Athens, Greece, 28-30 June 2021

\title{
AGGREGATED FORMULATION OF GEOMETRIC CONSTRAINTS FOR NODE-BASED SHAPE OPTIMIZATION WITH VERTEX MORPHING (EUROGEN 2021)
}

\author{
Armin Geiser, Ihar Antonau, and Kai-Uwe Bletzinger \\ Chair of Structural Analysis \\ Technical University of Munich \\ Arcisstr. 21, 80333 Munich, Germany \\ e-mail: $\{$ armin.geiser, ihar.antonau, kub\}@tum.de
}

\begin{abstract}
Node-based shape optimization methods like Vertex Morphing use every node of the surface mesh as design variables. The method's key advantages are the minimal effort for problem setup and the resulting largest possible design space that allows finding new and innovative shapes. Due to a large number of design variables, the application of gradient-based optimization methods becomes mandatory, and adjoint sensitivity analysis is the preferred way for efficient computation of the shape gradients.

For some industrial applications, it is necessary to restrict the large design freedom in some sense, for example by limiting the geometric design space. In recent work, nodal nonpenetration constraints have been successfully applied in the context of Vertex Morphing, resulting in a potentially large number of active constraints. Aggregation methods are commonly used to reduce the number of constraints in the optimization problem and are investigated here to combine nodal geometric constraints in a single global constraint function.
\end{abstract}

Keywords: Node-based, Shape Optimization, Packaging, Minimum Thickness, Geometric Constraints, Aggregation 


\section{INTRODUCTION}

Shape optimization is a powerful tool for speeding up the design process that has been proven to be useful in many disciplines. There is a vast number of different methods available in the literature, CAD- or IGA-based methods [1, 2], node-based methods [3, 4, 5, 6, 7] and level-set methods [8]. Vertex Morphing [9, 10, 11] is a parameterization method for node-based shape optimization. In node-based shape parametrizations, the surface nodes of the computational model are directly used as design parameters. No additional parametrization step is required to set up the optimization problem, and a high number of design parameters leads to the richest possible design space and provides the highest design potential. In practical applications, the rich design space can often not be explored freely but is geometrically limited by design requirements e.g. a packaging geometry. Such geometric constraints have to be considered in the optimization problem to avoid finding infeasible designs.

In the context of node-based shape optimization, geometric constraints for symmetry (implicit) and demolding (explicit) have been shown in [12]. Constraints for the thickness of solid models have been developed by [13] for level-set optimization, using a single aggregated constraint for efficiency reasons. Similarly, thickness constraints to control the minimum member size in node-based shape optimization have been realized by [14]. Nodal non-penetration constraints to satisfy packaging requirements have been introduced by [15] in shape optimization with the Vertex Morphing parametrization. Aggregation of nodal non-penetration constraints using a Kreisselmeier-Steinhausser function for FFD-based shape optimization was proposed by [16]. Another approach of aggregating nodal non-penetration constraints using a secondorder continuous penalty function was proposed by [17] and evaluated for spline-based shape parameterizations.

This paper is structured as follows: First, the Vertex Morphing parameterization is explained, followed by the gradient-projection method, which is used as an optimization algorithm in this work. Then, two geometric constraints, a packaging constraint and a minimum thickness constraint for node-based shape optimization are described and two constraint aggregation functions are presented. Finally, the application of the geometric constraints in combination with the aggregation functions is shown on two numerical examples with the Vertex Morphing parameterization, and their performance is evaluated in comparison with the node-wise application of the constraints.

\subsection{Vertex Morphing}

The basic theory of shape optimization with the Vertex Morphing parametrization is briefly introduced, the interested reader can refer to $[10,11]$ for more details.

Vertex Morphing introduces a design control field $p$ that controls the shape $z$ via convolution with a filter function $F$.

$$
z\left(\xi_{i}\right)=\int_{\xi_{i}-r}^{\xi_{i}+r} F\left(\xi_{i}, \xi, r\right) p d \xi
$$

Where $\xi_{i}$ is the surface coordinate of the unknown point and $r$ is the radius of the filter function. Accordingly, the shape update is controlled by the update of the control field in the same way.

The shape of the filter function is not as decisive, often linear hat functions are used, the radius has a major influence on the smoothness of the generated shape and shape update respectively. The filter radius can be seen as an additional design parameter, which controls the 
desired shape modes of the optimal design.

In discrete form, Equation 1 becomes

$$
z=B p
$$

and the vector of discrete shape coordinates $z$ is generated by multiplying the vector of control node coordinates $\boldsymbol{p}$ with the morphing matrix (or filter matrix) $\boldsymbol{B}$. $\boldsymbol{B}$ has a size of $m \times n$, with the $m$ being the number of shape coordinates $\boldsymbol{z}$ and $n$ the number of control coordinates $\boldsymbol{p}$. Usually, the same discretization is used for the shape and the design control field, but this is not mandatory.

A shape optimization problem with the Vertex Morphing parameterization can therefore be formulated as follows:

$$
\begin{array}{cl}
\min _{\boldsymbol{p}} & f(\boldsymbol{z}(\boldsymbol{p})) \\
\text { s.t. } & g_{j}(\boldsymbol{z}(\boldsymbol{p})) \leq 0, \quad j=1 \ldots n_{g}, \\
& h_{k}(\boldsymbol{z}(\boldsymbol{p}))=0, \quad k=1 \ldots n_{h}
\end{array}
$$

where $f(\boldsymbol{z}(\boldsymbol{p})$ is the objective function, the control point coordinates of Vertex Morphing $\boldsymbol{p}$ are the design variables, $g_{j}$ and $h_{k}$ are the inequality and equality constraints respectively. In gradient-based shape optimization, also the update of the design variables, as well as the design control gradient is of interest. It is straight forward to compute the discrete shape update $\Delta \boldsymbol{z}$ from the design control update $\Delta \boldsymbol{p}$ by applying Equation 2:

$$
\Delta z=B \Delta p
$$

The discrete design gradient of the objective $\nabla_{p} f$ is determined from the discrete shape gradient $\nabla_{z} f$ following the chain rule of differentiation by a so-called backward-mapping operation with the transpose of the morphing matrix

$$
\nabla_{p} f=\boldsymbol{B}^{T} \nabla_{z} f
$$

The gradients of the constraints are mapped accordingly.

\subsection{Optimization algorithm}

In this work, constraints are considered using an adaption of Rosen's gradient projection algorithm [18, 19] with the suggestions of Arora and Haug as described in [20]. The search direction $s$ is determined by projecting the objective gradient in the subspace tangent to the active constraints.

$$
s=\nabla_{p} f-\left[\boldsymbol{N}\left(\boldsymbol{N}^{\boldsymbol{T}} \boldsymbol{N}\right)^{-1} \boldsymbol{N}^{\boldsymbol{T}}\right] \nabla_{p} f
$$

where $\boldsymbol{N}$ is the matrix of the active constraint gradients with a size of $n \times k$, with the number of active constraints $k$ and the number of design variables $n$.

For nonlinear constraints, this projection step can still lead to a violation because the curvature of the constraint is not considered. To avoid a drift-off error throughout the optimization, this violation has to be corrected. In this work, the correction is not made immediately, but in 


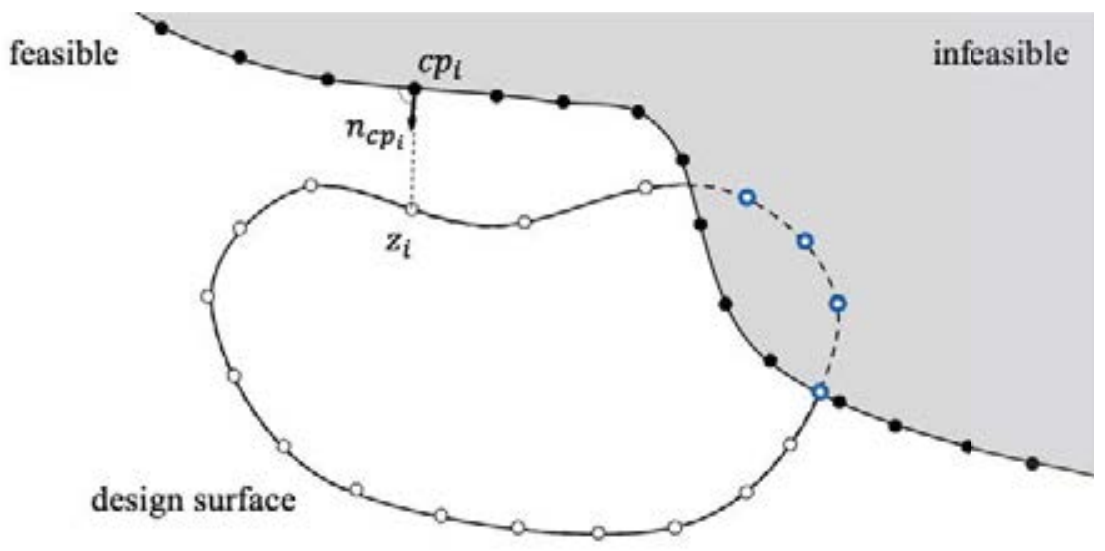

Figure 1: Nodal packaging constraint. Infeasible nodes are highlighted in blue.

the next optimization step, to avoid multiple evaluations of the constraint functions. It is determined by the vector of the active constraint values $\boldsymbol{g}_{\boldsymbol{a}}$ and the matrix of constraint gradients $N$ :

$$
c=-\boldsymbol{N}\left(\boldsymbol{N}^{\boldsymbol{T}} \boldsymbol{N}\right)^{-1} \boldsymbol{g}_{\boldsymbol{a}}
$$

The update of the control parameters is finally computed by combining the search direction and the correction.

$$
\Delta \boldsymbol{p}=\alpha \boldsymbol{s}+\boldsymbol{c}
$$

$\alpha$ is the step length factor and can be determined by a line-search. For practical applications and in this work, we limit the shape update in each iteration by applying an upper limit for $\|\alpha \boldsymbol{s}\|^{\infty}$ and $\|\boldsymbol{c}\|^{\infty}$ individually. [15] has successfully used the same projection algorithm with a constant step length for imposing the nodal non-penetration constraints, however, without the correction therm in Equation 6. [21, 22] have developed alternative optimization algorithms that have proven to work well with the Vertex Morphing parametrization.

\section{GEOMETRIC CONSTRAINTS}

The path of the design nodes through the design space during the shape optimization with Vertex Morphing is not known a priori. Therefore it is often not possible to describe the geometrical limitation of the design space by static variable bounds. Instead, constraints can be added to the optimization problem. Two types of geometric constraints are presented in this section.

\subsection{Packaging constraint}

A packaging constraint geometrically limits the design space by dividing it into a feasible and infeasible domain. Figure 1 shows a shape optimization problem with the discrete design surface described by the mesh nodes with coordinates $z_{i}$ and a discrete packaging geometry. The surface normals of the packaging geometry point to the feasible domain. The boundary of the infeasible domain can be represented using arbitrary geometries, which leads to maximal flexibility in the setup of the optimization problem.

The design surface is not allowed to penetrate the packaging geometry, this can be formulated by node-wise inequality constraints [15]. The value of the packaging constraint at node $z_{i}$ is 

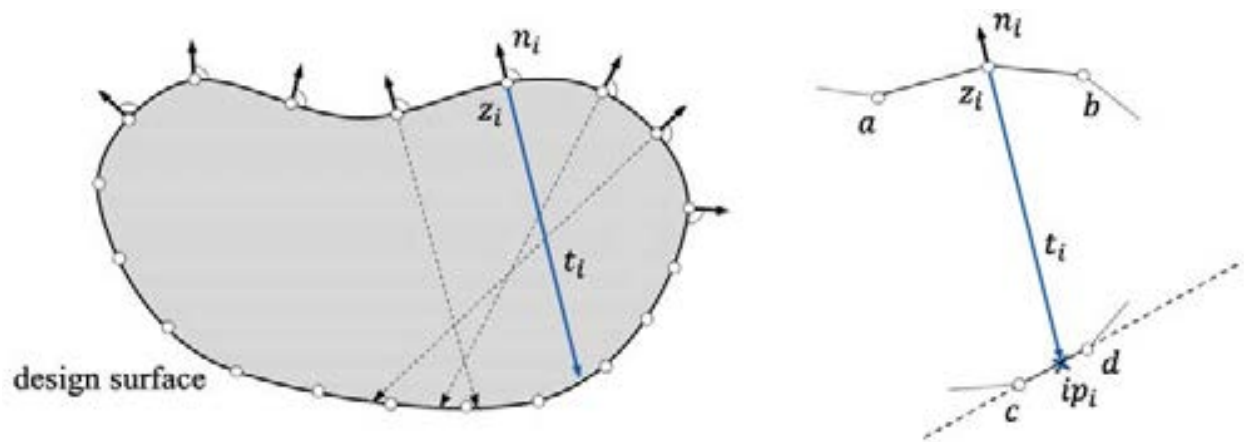

Figure 2: Detection of local thickness with ray-surface intersection on discrete surfaces. The sketch on the right shows the nodes that affect the local thickness vector at node $\boldsymbol{t}_{\boldsymbol{i}}$

determined by the distance vector to the closest point $\boldsymbol{c} \boldsymbol{p}_{\boldsymbol{i}}$ on the packaging geometry, and the surface unit normal $\boldsymbol{n}_{\boldsymbol{c} p_{i}}$ of this closest point.

$$
g_{i}=\left(\boldsymbol{z}_{\boldsymbol{i}}-\boldsymbol{c} \boldsymbol{p}_{\boldsymbol{i}}\right)^{T} \boldsymbol{n}_{\boldsymbol{c} \boldsymbol{p}_{\boldsymbol{i}}} \leq 0
$$

$g_{i}$ is positive if node $\boldsymbol{z}_{\boldsymbol{i}}$ penetrates the design boundary, zero if it lies on the boundary, and negative if it is in the feasible domain.

In this work, detection of the closest point on the packaging boundary is done using a nearestneighbor search. For practical applications, this appears to be accurate enough if the discretization of the packaging geometry is fine enough.

The gradient of the response function is computed under the assumption, that the closest point will not change due to a movement of the point $z_{i}$. In this case, the nodal gradient is the normal of the closes point at the position $i$ and 0 elsewhere.

$$
\nabla_{z} g_{i}=\left[0 \ldots \boldsymbol{n}_{\boldsymbol{c} \boldsymbol{p}_{\boldsymbol{i}}} \ldots 0.0\right]^{T}
$$

\subsection{Minimum thickness constraint}

A limitation of the minimum thickness of the geometry is another geometric constraint that is often required. Figure 2 shows a shape optimization problem with the discrete design surface described by the mesh nodes $z_{i}$ and the definition of a nodal thickness value that is used in this work. The nodal thickness is determined by following a ray in negative surface normal direction $-\boldsymbol{n}_{\boldsymbol{i}}$ until an intersection $\boldsymbol{i} \boldsymbol{p}_{\boldsymbol{i}}$ with the design surface is found. This operation has been realized in a numerically efficient way with an octree-based ray-element intersection algorithm. With this approach, only a surface discretization is required and no discretization of the solid is needed.

The value of the thickness constraint at node $z_{i}$ is determined by the difference of the limit value for the thickness $t_{\min }$ and the norm of the nodal thickness vector $\boldsymbol{t}_{\boldsymbol{i}}$.

$$
g_{i}=t_{\text {min }}-\left\|\boldsymbol{z}_{\boldsymbol{i}}-\boldsymbol{i} \boldsymbol{p}_{\boldsymbol{i}}\right\|=t_{\text {min }}-\left\|\boldsymbol{t}_{\boldsymbol{i}}\right\| \leq 0
$$

The thickness response value is affected by several nodes as can be seen in Figure 2 on the right side for the 2-dimensional case. The node $z_{i}$, the direct neighbors $(a, b)$ of $z_{i}$, since they affect the orientation of the surface normal, and the nodes of the element that is intersected by the ray $(c, d)$. The gradient of the thickness response function is computed under the assumption, that the ray will not intersect another element than initially detected $(\overline{c d})$. The same logic holds for surfaces in 3 dimensions. 
[13] formulated thickness constraints for level-set optimization based on a medial axis defined by a skeleton model. [14] has also formulated a minimum thickness constraint based on an approximated medial axis and considered the medial axis to be fixed in the computation of the gradients.

\section{AGGREGATION OF NODAL GEOMETRIC CONSTRAINTS}

The node-wise formulation of geometric constraints in the previous section can lead to a high number of active constraints in the optimization problem that is in the order of the number of design variables [13]. For shape optimization with Vertex Morphing, the constraint gradients are consistently mapped to the control space (Equation 4). The contribution of the nodal constraints to the matrix $N$ has a sparse pattern, however, the bandwidth is determined by the filter radius and can be large. For large models with a high number of design variables in combination with a large filter radius, the solution of the equation system in equations 5,6 can become numerically challenging [15]. An alternative to considering each nodal constraint individually is the aggregation of the individual nodal constraints into a single global constraint function. This allows satisfying the nodal constraints in an average sense and numerically less expensive way. [13, 16, 17] have previously applied aggregation formulations for geometric constraints in shape optimization. Constraint aggregation is also commonly used for stress constraints in structural optimization see e.g. [23]. In this work, two aggregation functions are evaluated for nodal geometric constraints in node-based shape optimization with the Vertex Morphing parameterization.

One possibility to aggregate the nodal constraints is to take the 2-norm of all nodal constraints that are infeasible, in other words, have a positive nodal constraint value $g_{i}^{+}$(Equation 8).

$$
g_{2 \text {-norm }}=\sqrt{\sum_{i=1}^{n}\left(g_{i}^{+}\right)^{2}}
$$

Since only infeasible nodes contribute to the aggregated constraint function $g_{2 \text {-norm }}$, only gradients of the active constraints contribute to the gradient of the aggregated function. It is not possible to foresee if a node will soon violate the constraint. New nodes can suddenly become infeasible, potentially leading to a zig-zagging behavior. This makes the correction step (Equation 6) important.

As an alternative to the aggregation using a 2-norm, a Kreisselmeier-Steinhausser (KS) function [24] might be used. In contrast to the aggregation of only infeasible values with the 2-norm, the Kreisselmeier-Steinhausser function takes all nodal values into account.

$$
g_{\mathrm{KS}}=\frac{1}{\rho} \ln \sum_{i=1}^{n} e^{\left(\rho g_{i} / \beta\right)}
$$

The function $g_{\mathrm{KS}}\left(g_{i}\right)$ approximates the non-continuous max-function $\max \left(g_{i}\right)$. The factor $\beta$ is used to bring the nodal constraint values $g_{i}$ to a common scale, independent of model units. In this work, we use the maximum allowed shape update for $\beta$. The approximation becomes more accurate with higher values of $\rho$, too high values can lead to numerical overflow, however. All nodes, except the one with the highest constraint value, contribute very little to the aggregated gradient, this can lead to a similar problem with nodes suddenly becoming infeasible as for the 2-norm aggregation. Smaller values for $\rho$ lead to a more conservative approximation of the 
maximum violation. This results in a smoother behavior of the constraint in the optimization but eventually does not allow the optimization to converge close to the actual limit of the nodal constraints. This effect will be demonstrated in the following numerical example.

\section{NUMERICAL EXAMPLE}

A simple 1D example is used to describe the basic effects of constraint aggregation for geometric constraints in shape optimization with the Vertex Morphing parametrization. The deviation of an initially straight line to a hat-shaped target curve with a height of 1.0 is minimized, while an obstacle has to be considered.

$$
\min _{\boldsymbol{p}} f(\boldsymbol{z}(\boldsymbol{p}))=\sum_{i=1}^{n}\left(z_{i}(\boldsymbol{p})-z_{i}^{\text {hat }}\right)^{2}
$$

with the target function $z^{\text {hat }}$ :

$$
z^{h a t}(\xi)= \begin{cases}0 & \xi \leq-10 \\ \frac{\xi}{10}+1 & -10 \leq \xi \leq 0 \\ \frac{-\xi}{10}+1 & 0 \leq \xi \leq 10 \\ 0 & 10 \leq \xi\end{cases}
$$

The line is discretized with a mesh size of 0.5 and $n=81$ nodes. A Vertex Morphing filter radius $r=5.0$ is used. The obstacle geometry is discretized with the same mesh size and limits the vertical extension of the design line. The shape optimization is executed for 100 iterations using the gradient-projection algorithm (subsection 1.2) with an upper limit on the step length and correction of 0.01 . The optimization is executed with the aggregation functions and compared with a reference solution using nodal constraints.

- 2-norm aggregation: $g_{2 \text {-norm }} \leq 0$

- Kreisselmeier-Steinhausser: $g_{\mathrm{KS}}(\rho=10) \leq 0$

- Kreisselmeier-Steinhausser: $g_{\mathrm{KS}}(\rho=30) \leq 0$

- nodal constraints: $g_{i} \leq 0$ for $i=1 \ldots n$ nodes

All four examples converge to almost exactly the same final shape - a smooth representation of the target hat-function respecting the design boundary, as shown in Figure 3.

The objective convergence for all cases is very similar (Figure 4a). Looking at the maximum nodal violation of the packaging constraint in Figure $4 \mathrm{~b}$ reveals oscillations for the case with 2-norm aggregation. These oscillations are caused by nodes that jump between the feasible and infeasible domain over the optimization iterations. This is to be expected for the 2-norm aggregation since feasible nodes do not contribute at all to the aggregated response gradient. The oscillations between steps 25 and 60 occur at the center of the line, where the bump in the packaging geometry is hit first by the design geometry. Here, a maximum nodal violation in the order of the maximum shape update occurs if the previous design was feasible. This violation is corrected in the following iterations until the design becomes feasible again and the process repeats. After iteration 60, the design also hits the other part of the packaging 

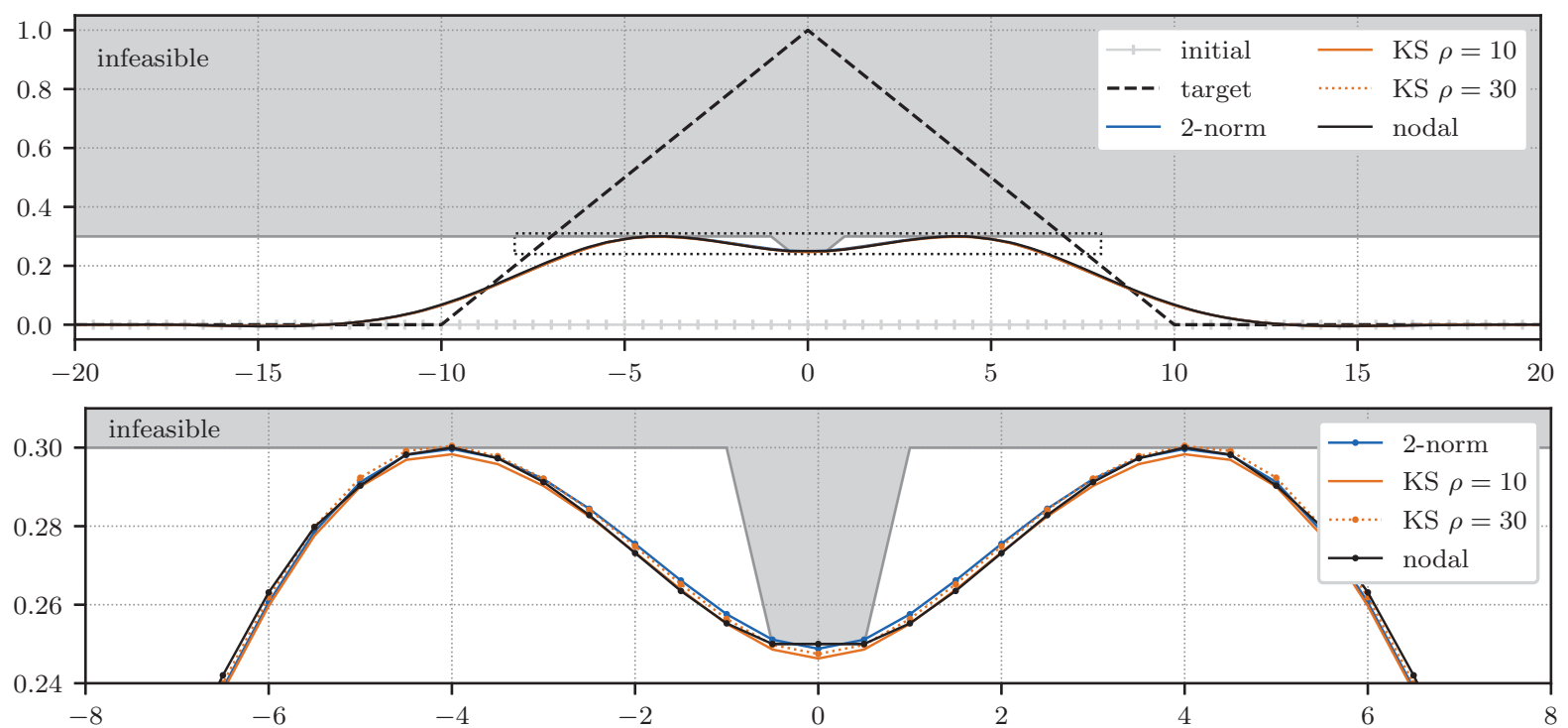

Figure 3: Final shape after 100 iterations using the different formulations for the packaging constraint and close-up near the obstacle

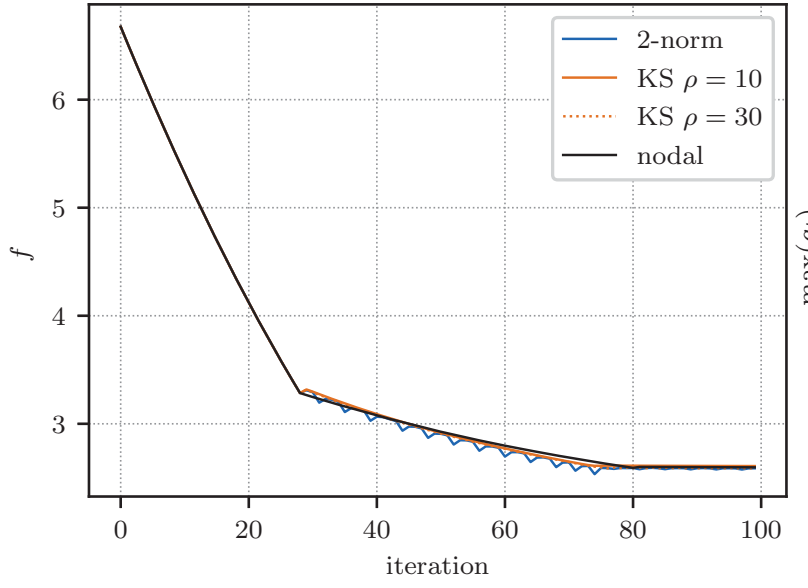

(a)

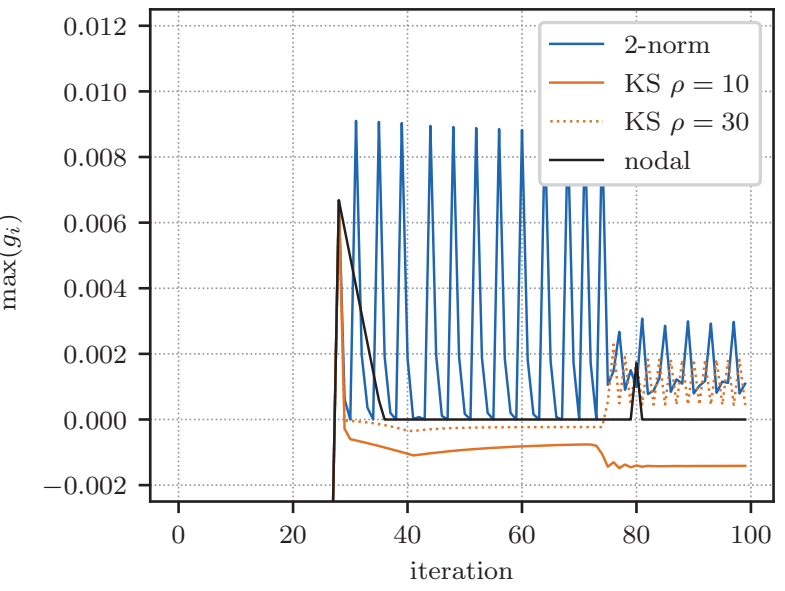

(b)

Figure 4: (a) Convergence graph of the objective function and (b) maximal nodal violation of the bounding geometry in each iteration. 
geometry at a height of 0.3 . From this point, the maximum nodal violation jumps between the different geometric locations of the contact points with the packaging geometry. The aggregated constraint stays active all the time, however, individual nodes still oscillate between feasible and infeasible domains. The overall maximum violation does not vanish even after many iterations, however, it remains bounded by the step size.

The case with Kreisselmeier-Steinhausser aggregation with $\rho=30$ shows no oscillations until iteration 60, where the shape hits the packaging geometry at tree locations. From this point, oscillations of the maximum nodal violation are observed, similar to the case with the 2-norm aggregation. This can be explained by the fact, that high $\rho$ values result in a good approximation of the maximum value. The gradient of the aggregated constraint is dominated by the gradient of the node with the maximum violation, and even if all other nodes still contribute, they are underrepresented, and the computed shape update might push them to the infeasible domain. The smaller value for $\rho=10$ in the Kreisselmeier-Steinhausser aggregation leads to a more conservative approximation of the maximum violation. The contribution of the nodes to the aggregated response value and gradient is more evenly distributed and relaxes the zig-zagging behavior. This comes with a price, the approximation is too conservative and the design geometry is constrained with a distance to the packaging geometry. An optimal choice of $\rho$ is problem-dependent, since the number of design variables and therefore nodal constraints, and the number of nodes that come close to the boundary affect the behavior.

The reference solution with the nodal constraints does not show any oscillatory behavior and the packaging constraint is satisfied exactly.

In general, all four cases satisfy the geometric constraint, with small violations for the aggregation with the 2-norm and the Kreisselmeier-Steinhausser function with $\rho=30$. Such small violations of geometric constraints are often acceptable, especially if Vertex Morphing is used to find new shapes in early design stages.

\section{STRUCTURAL OPTIMIZATION EXAMPLE}

As a second application example, we present a structural optimization of a solid hook model. The hook has a height of $237 \mathrm{~mm}$ and is modeled with a linear elastic material with a Young's modulus $E=206.9 \mathrm{GPa}$ and a Poisson's ratio $\nu=0.29$. The mass of to hook should be minimized while maintaining the initial compliance for two static load cases with a load at the center (LC1: $\mathrm{F}=32 \mathrm{kN}$ ) and tip (LC2: $\mathrm{F}=16 \mathrm{kN})$ respectively. For both load cases, the hook is supported at the top. In addition to the structural constraints, two geometric constraints from the previous sections are applied. The design space is limited by a curved packaging geometry with 4505 nodes, and a minimum thickness constraint of $t_{\min }=15.0 \mathrm{~mm}$. The load application area and the supports are excluded from the shape optimization, this leads to a total number of 7117 surface nodes in the design area. A smooth transition is enforced between design and non-design regions. The setup of the optimization problem with the initial shape and discretization of hook and packaging geometry is shown in Figure 5. A filter radius of $r=25.0 \mathrm{~mm}$ is used for the Vertex Morphing parameterization, the maximum step length in search direction and correction is limited to $1.0 \mathrm{~mm}$. The shape optimization, including the adjoint sensitivity analysis of the structural response functions and the pseudo-elastic mesh motion for the internal nodes, is done with the ShapeOptimization-, StructuralMechanics-, and MeshMovingApplication of the opensource software Kratos-Multiphysics [25]. The optimization is stopped after 100 iterations.

Three cases of the optimization problem are solved, comparing the different formulations for the geometric constraints: 

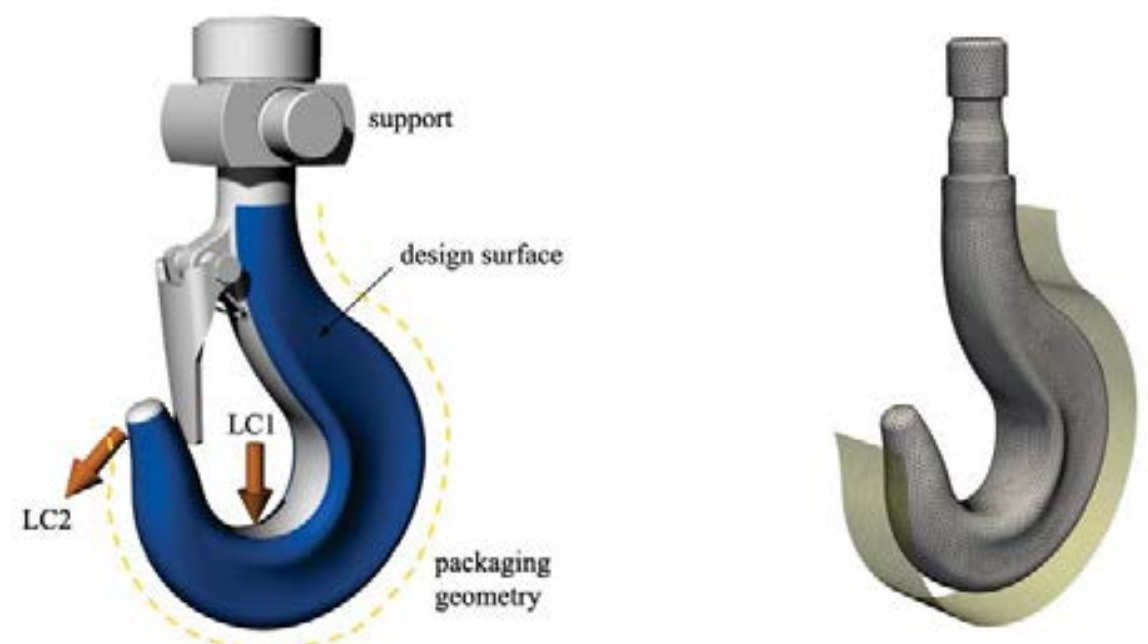

Figure 5: Optimization setup of the hook with the load-cases, the design surface (blue), the packaging geometry (yellow) and the simulation meshes.
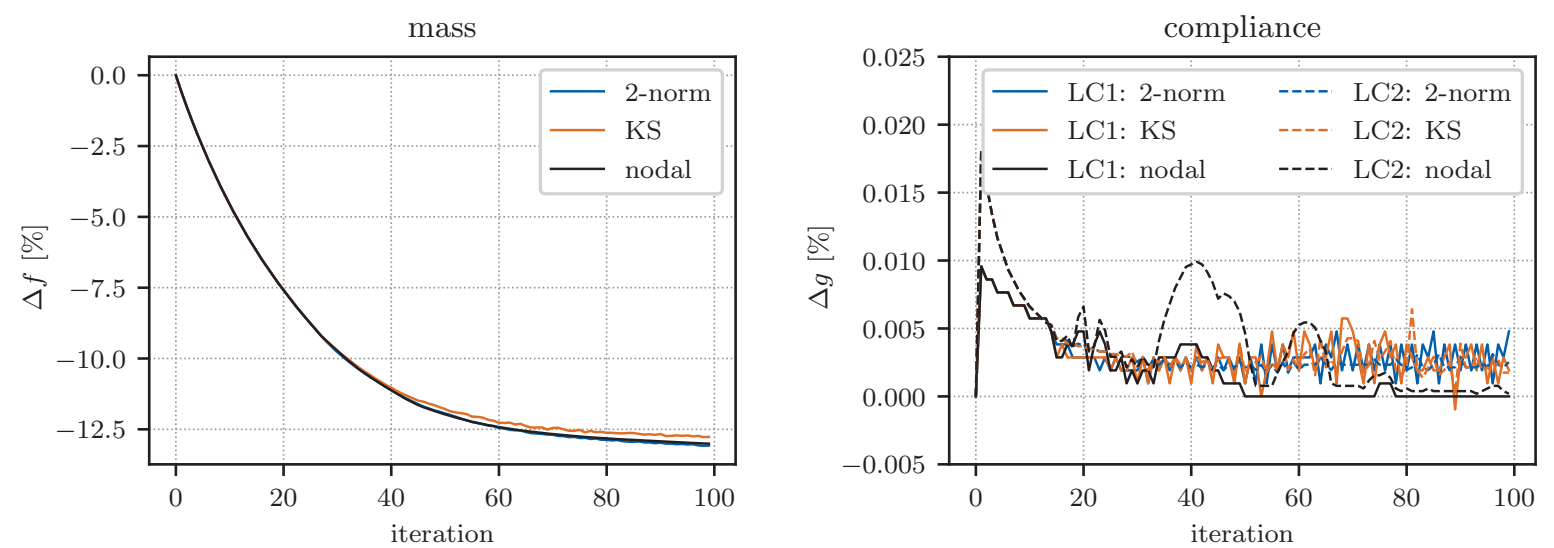

Figure 6: Improvement of mass objective and violation of the compliance constraints for the three cases during the optimization process.

- 2-norm aggregation: $g_{2 \text {-norm }} \leq 0$

- Kreisselmeier-Steinhausser: $g_{\mathrm{KS}}(\rho=18) \leq 0$

- nodal constraints: $g_{i} \leq 0$ for $i=1 \ldots n$ nodes

The shape optimization converges to very similar shapes for all three cases, with a mass reduction of $\approx 13 \%$. The case with the Kreisselmeier-Steinhausser function leads to a bit less improvement due to the conservative approximation of the maximum nodal violation for the geometric constraints (Figure 6 left). Even though the aggregated geometric constraints cause slight oscillations for the objective and the compliance constraints, the overall trend is comparable to the case with node-wise constraints. The structural constraints are active during the whole optimization, they are violated by less than $0.005 \%$ (Figure 6 right) for all three cases, which is negligible.

Figure 7 shows the maximum nodal violation of the packaging constraint and the minimum thickness constraint respectively for the three cases. The 2-norm aggregation again leads to a 

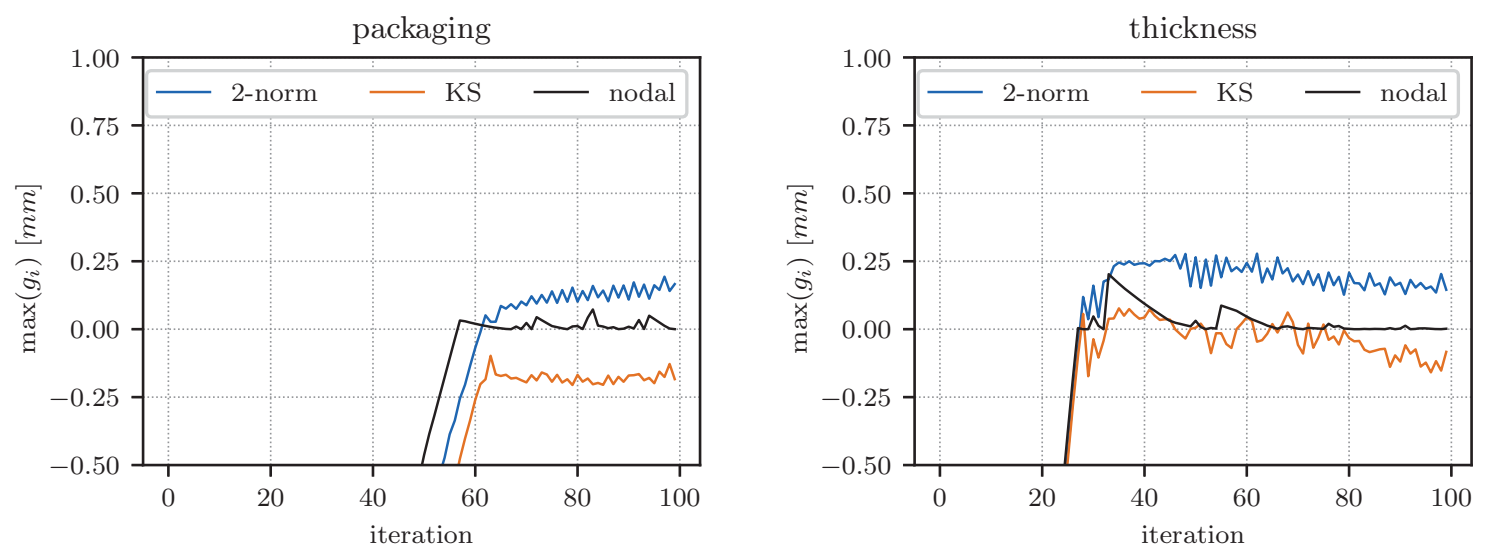

Figure 7: Maximum value of all nodal violations of packaging and thickness constraints for the three cases during the optimization process. The violations remain much smaller then the applied step size.
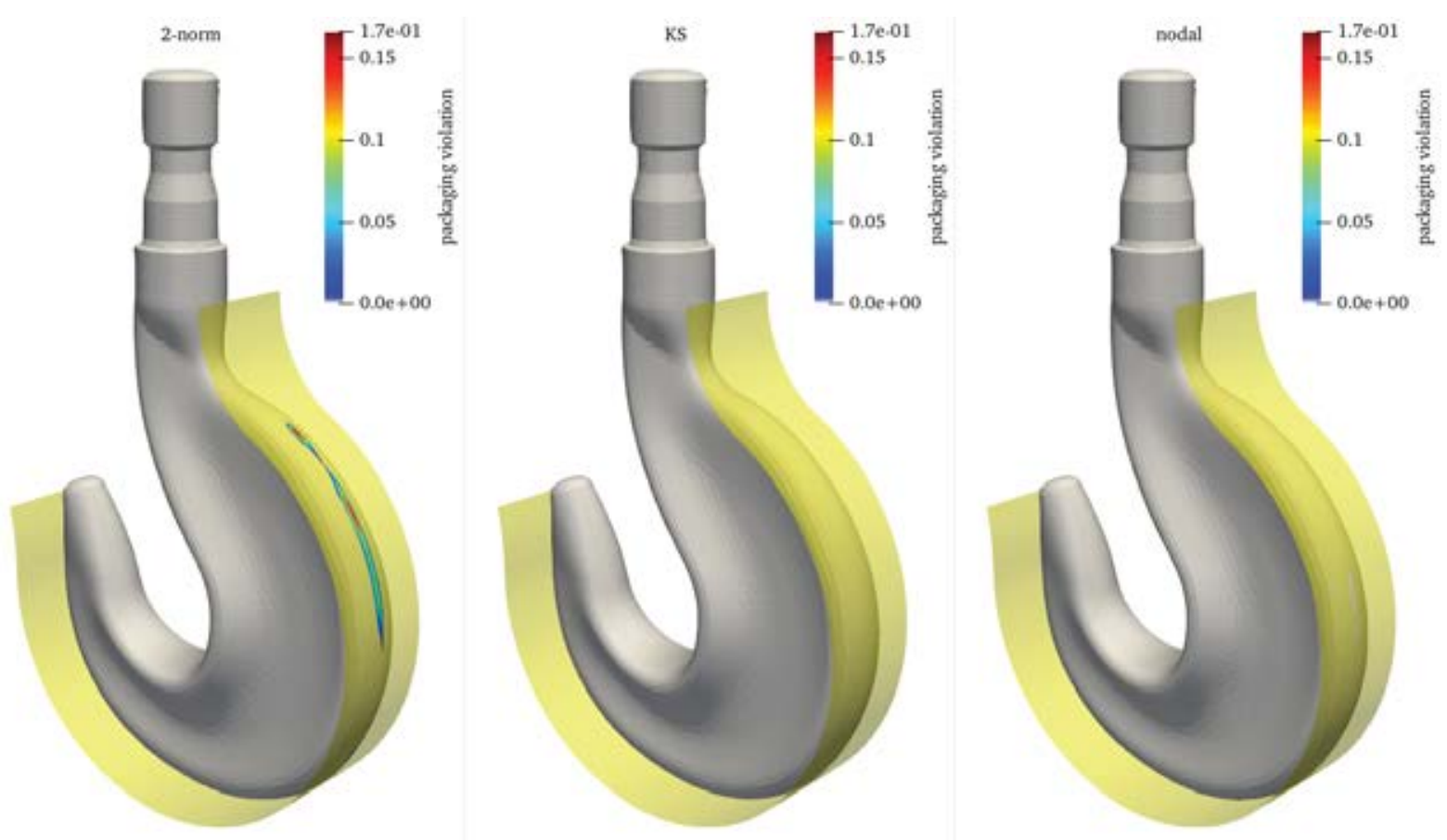

Figure 8: Final shape of the hook for the three cases, from left to right: 2-norm aggregation, Kreisselmeier-Steinhausser aggregation, nodal constraints. The nodal violation of the packaging constraint is colored, feasible nodes are colorless. 

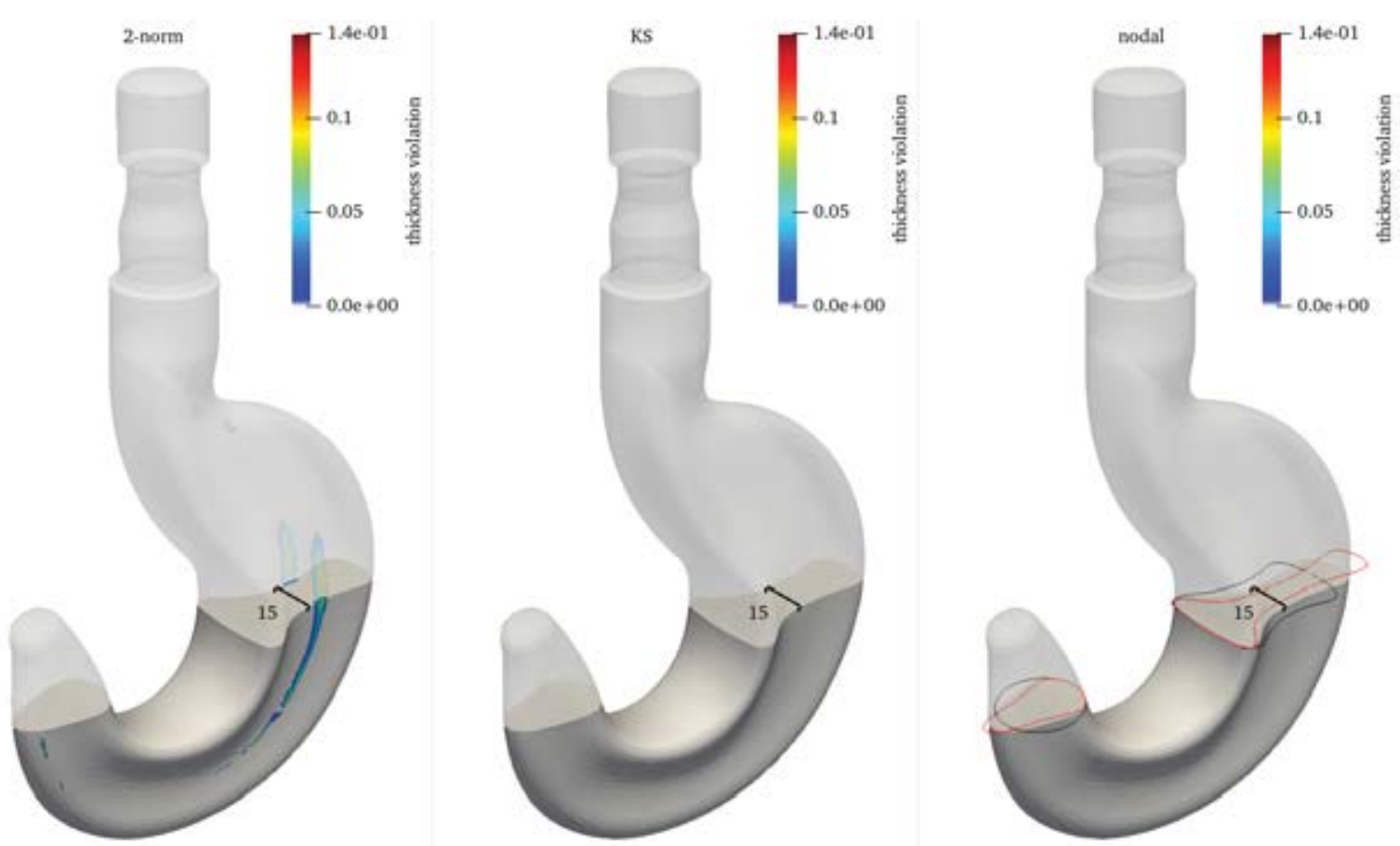

Figure 9: Final shape of the hook optimization showing the nodal violation of the thickness constraint. Feasible nodes are colorless. In the right figure, the initial (black) and geometrically unconstrained final shape (red) are indicated.

zig-zagging behavior of the nodal violation and finally results in a maximum violation of $\approx 0.2$ $\mathrm{mm}$ of both geometric constraints. Individual nodes that violate the constraint are pushed to the feasible domain by the correction step after one iteration. Those nodes do not contribute to the aggregated constraint gradient anymore and jump to the infeasible domain in the following iteration. The maximum nodal value $g_{i}$ does not occur at the same node in each iteration. The Kreisselmeier-Steinhausser formulation is able to satisfy the geometric constraint with slightly smaller oscillations but is more conservative. The design surface is stopped within a distance of $\approx 0.2 \mathrm{~mm}$ to the packaging geometry and $\approx 0.1 \mathrm{~mm}$ to the minimum thickness constraint. This leads to a slightly smaller objective improvement for this case. With another choice of $\rho$ this might be improved, however, i.e. $\rho=25$ already leads to much larger oscillations. The case with the nodal formulation is again able to satisfy the packaging and also the minimum thickness constraints exactly, as expected. The final shapes with the nodal violations of the packaging constraint are shown in Figure 8. The nodal violations of the thickness constraint are shown in Figure 9.

This example demonstrates that aggregation of geometric constraints can be applied also in more complex shape optimization problems when other physical constraints have to be considered. The maximum number of simultaneously active constraints in this example is 173 for the node-wise constraints and 4 for the aggregated formulations. Even though the aggregation of the geometric constraints does amplify the known zig-zagging behavior of the gradient-projection algorithm, the negative impact on the objective improvement and enforcement of the physical constraints is negligible. It is highlighted that globally the same shapes are found in all three cases. 


\section{CONCLUSIONS}

Constraint aggregation functions have been successfully applied to geometric constraints in shape optimization problems with the Vertex Morphing parameterization and a gradientprojection algorithm. The performance of the aggregation functions has been evaluated in an academic 1D example and a 3D structural-mechanics example, comparing with the node-wise application of the constraints. The latter example was also subject to additional structural constraints. The overall number of constraints can be drastically reduced, which is of interest, especially for large models. With both investigated constraint aggregation functions, the same shapes as are found as with the reference solution with nodal geometric constraints. Depending on the aggregation formulation, the final shape does either slightly violate the geometric constraint, or is stopped within a small distance to the constraint limit in case of a conservative choice of the $\rho$ value of the Kreisselmeier-Steinhausser formulation. Such small deviations from the geometric constraints are often acceptable when searching new shapes, and constraint aggregation can be an efficient alternative to the numerically challenging nodal application of the constraints.

\section{ACKNOWLEDGEMENT}

This work has been carried out within the MADELEINE project which has received funding from the European Union's Horizon 2020 research and innovation programme under grant agreement N 769025.

\section{References}

[1] Shenren Xu, Wolfram Jahn, and Jens-Dominik Müller. CAD-based shape optimisation with CFD using a discrete adjoint. International Journal for Numerical Methods in Fluids, 74(3):153-168, 2014. ISSN 1097-0363. doi: 10.1002/fld.3844.

[2] J. Kiendl, R. Schmidt, R. Wüchner, and K.-U. Bletzinger. Isogeometric shape optimization of shells using semi-analytical sensitivity analysis and sensitivity weighting. Computer Methods in Applied Mechanics and Engineering, 274:148-167, June 2014. ISSN 00457825. doi: 10.1016/j.cma.2014.02.001.

[3] Antony Jameson. Optimum aerodynamic design using CFD and control theory. In 12th Computational Fluid Dynamics Conference, San Diego,CA,U.S.A., June 1995. American Institute of Aeronautics and Astronautics. doi: 10.2514/6.1995-1729.

[4] Hideyuki Azegami and Kenzen Takeuchi. A smoothing method for shape optimization: Traction method using the Robin condition. International Journal of Computational Methods, 03(01):21-33, March 2006. ISSN 0219-8762, 1793-6969. doi: $10.1142 / \mathrm{S} 0219876206000709$.

[5] Chau Le, Tyler Bruns, and Daniel Tortorelli. A gradient-based, parameter-free approach to shape optimization. Computer Methods in Applied Mechanics and Engineering, 200 (9-12):985-996, February 2011. ISSN 00457825. doi: 10.1016/j.cma.2010.10.004.

[6] Stefan Riehl, Jan Friederich, Michael Scherer, Ralf Meske, and Paul Steinmann. On the discrete variant of the traction method in parameter-free shape optimization. Computer Methods in Applied Mechanics and Engineering, 278:119-144, August 2014. ISSN 00457825. doi: 10.1016/j.cma.2014.05.009. 
[7] Matthias Firl and Kai-Uwe Bletzinger. Shape optimization of thin walled structures governed by geometrically nonlinear mechanics. Computer Methods in Applied Mechanics and Engineering, 237-240:107-117, September 2012. ISSN 0045-7825. doi: 10.1016/j.cma.2012.05.016.

[8] Grégoire Allaire, François Jouve, and Anca-Maria Toader. A level-set method for shape optimization. Comptes Rendus Mathematique, 334(12):1125-1130, January 2002. ISSN 1631-073X. doi: 10.1016/S1631-073X(02)02412-3.

[9] Kai-Uwe Bletzinger. A consistent frame for sensitivity filtering and the vertex assigned morphing of optimal shape. Structural and Multidisciplinary Optimization, 49(6):873895, June 2014. ISSN 1615-147X, 1615-1488. doi: 10.1007/s00158-013-1031-5.

[10] Majid Hojjat, Electra Stavropoulou, and Kai-Uwe Bletzinger. The Vertex Morphing method for node-based shape optimization. Computer Methods in Applied Mechanics and Engineering, 268:494-513, January 2014. ISSN 00457825. doi: 10.1016/j.cma.2013. 10.015 .

[11] Kai-Uwe Bletzinger. Shape Optimization. In Erwin Stein, René de Borst, and Thomas J R Hughes, editors, Encyclopedia of Computational Mechanics Second Edition, pages 142. John Wiley \& Sons, Ltd, Chichester, UK, December 2017. ISBN 978-1-119-00379-3 978-1-119-17681-7. doi: 10.1002/9781119176817.ecm2109.

[12] Oliver Schmitt, Jan Friederich, Stefan Riehl, and Paul Steinmann. On the formulation and implementation of geometric and manufacturing constraints in node-based shape optimization. Structural and Multidisciplinary Optimization, 53(4):881-892, April 2016. ISSN 1615-1488. doi: 10.1007/s00158-015-1359-0.

[13] G. Allaire, F. Jouve, and G. Michailidis. Thickness control in structural optimization via a level set method. Structural and Multidisciplinary Optimization, 53(6):1349-1382, June 2016. ISSN 1615-1488. doi: 10.1007/s00158-016-1453-y.

[14] Oliver Schmitt and Paul Steinmann. Control of minimum member size in parameterfree structural shape optimization by a medial axis approximation. Computational Mechanics, 61(6):717-727, June 2018. ISSN 0178-7675, 1432-0924. doi: 10.1007/ s00466-017-1477-1.

[15] Reza Najian Asl, Shahrokh Shayegan, Armin Geiser, Majid Hojjat, and Kai-Uwe Bletzinger. A consistent formulation for imposing packaging constraints in shape optimization using Vertex Morphing parametrization. Structural and Multidisciplinary Optimization, 56(6):1507-1519, December 2017. ISSN 1615-147X, 1615-1488. doi: $10.1007 / \mathrm{s} 00158-017-1819-9$.

[16] Benjamin J. Brelje, Joshua L. Anibal, Anil Yildirim, Charles A. Mader, and Joaquim R. R. A. Martins. Flexible Formulation of Spatial Integration Constraints in Aerodynamic Shape Optimization. AIAA Journal, 58(6):2571-2580, May 2020. ISSN 0001-1452. doi: 10.2514/1.J058366.

[17] Marios Damigos, E. Papoutsis-Kiachagias, and Kyriakos Giannakoglou. Adjoint Variablebased Shape Optimization with Bounding Surface Constraints. International Journal for Numerical Methods in Fluids, 93, July 2020. doi: 10.1002/fld.4900. 
[18] J. B. Rosen. The Gradient Projection Method for Nonlinear Programming. Part I. Linear Constraints. Journal of the Society for Industrial and Applied Mathematics, 8(1):181-217, March 1960. ISSN 0368-4245, 2168-3484. doi: 10.1137/0108011.

[19] J. B. Rosen. The Gradient Projection Method for Nonlinear Programming. Part II. Nonlinear Constraints. Journal of the Society for Industrial and Applied Mathematics, 9(4): 514-532, December 1961. ISSN 0368-4245. doi: 10.1137/0109044.

[20] Raphael T. Haftka and Zafer Gürdal. Constrained Optimization. In Raphael T. Haftka and Zafer Gürdal, editors, Elements of Structural Optimization, Solid Mechanics And Its Applications, pages 159-207. Springer Netherlands, Dordrecht, 1992. ISBN 978-94-0112550-5. doi: 10.1007/978-94-011-2550-5_5.

[21] Long Chen, Kai-Uwe Bletzinger, Armin Geiser, and Roland Wüchner. A modified search direction method for inequality constrained optimization problems using the singularvalue decomposition of normalized response gradients. Structural and Multidisciplinary Optimization, 60(6):2305-2323, December 2019. ISSN 1615-147X, 1615-1488. doi: 10.1007/s00158-019-02320-9.

[22] Ihar Antonau, Majid Hojjat, and Kai-Uwe Bletzinger. Relaxed gradient projection algorithm for constrained node-based shape optimization. Structural and Multidisciplinary Optimization, February 2021. ISSN 1615-147X, 1615-1488. doi: 10.1007/ s00158-020-02821-y.

[23] Andrew B. Lambe, Graeme J. Kennedy, and Joaquim R. R. A. Martins. An evaluation of constraint aggregation strategies for wing box mass minimization. Structural and Multidisciplinary Optimization, 55(1):257-277, January 2017. ISSN 1615-147X, 1615-1488. doi: 10.1007/s00158-016-1495-1.

[24] G. Kreisselmeier and R. Steinhauser. Systematic Control Design by Optimizing a Vector Performance Index. IFAC Proceedings Volumes, 12(7):113-117, September 1979. ISSN 1474-6670. doi: 10.1016/S1474-6670(17)65584-8.

[25] Vicente Mataix Ferrándiz, Philipp Bucher, Riccardo Rossi, Rubén Zorrilla, Jordi Cotela, Josep Maria, Miguel Angel Celigueta, and Guillermo Casas. KratosMultiphysics/Kratos: KratosMultiphysics 8.1. Zenodo, November 2020. 